\title{
Digital Image Information Hiding Technology Based on Lifting Wavelet Transform and Image Fusion
}

\author{
Lei Yun \\ Jiangxi University of Science and Technology, Ganzhou 341000, China \\ yxly126@126.com
}

\begin{abstract}
A color multi-focus image fusion of self-feedback algorithm based on lifting wavelet transforms. Firstly, the basic principle of lifting wavelet transform is discussed. Secondly, considered feature of human vision system, the color images are transformed into YIQ color space and different fusion rules are used. The fusion parameters are substituted by extended chaotic sequences, this rule is used in fusing me and Q components, and an optimized fusion result of Y component is obtained by self-feedback fusion rule, then the three fusion results are transformed into RGB space. Based on the image fusion, we present a digital image hiding technology for images which have different size. In the process of image fusion, the fusion parameters are substituted by extended chaotic sequences, this approach makes the algorithms safer, and the experimental results indicate the algorithm has good efficiency and safety.
\end{abstract}

Keywords: Image fusion; Lifting wavelet transform; Image hiding; Chaotic sequences.

\section{Introduction}

As to color image fusion, the traditional multi-scale decomposition fusion method whose computation speed is slow and demand of memory is high, which is not suitable for the real-time application. So the passage puts forward the Color image fusion algorithm based on lifting wavelet transform [1]. It not only applies to improve algorithm in the superiority of computation time, but also adopts the way of feed-back to deal with the Intensity component to which the eyes are most sensitive based on feature of human visual sense, and meanwhile handles the color components by average method in order to reduce the damage in the fusion results of spectral information. This essay, on the basis of improving the image fusion method, shows a way of image hiding, which can deal with the digital image of the same size. It is proved that the method has better effect and safety.

\section{Digital Image Information Hiding based on Image Fusion}

The passage takes the characteristics of human visual sense into consideration, additionally involving the physical meanings of the YIQ three components. It also comes up with different fusion rules for Y brightness and IQ color components, and especially for the fusion process of the $\mathrm{Y}$ brightness components, a new way is presented. The contrite implementing process:

First: Because the RGB space is very uneven perceptually, two original color images are transferred from RGB color space to YIQ color space to obtain a representation more suitable to the characteristics of human visual sense [2].

Second: first of all deal with the IQ component, lifting wavelet transform to the IQ two colors components which belong to the two original pictures, and then fuse them with Chaotic sequences interpolation [3].

Here chaotic sequences can be structured by Logistic mapping; the chaotic system can be demonstrated:

$\mathrm{ak}+1=\mu \cdot \mathrm{ak} \cdot(1-\mathrm{ak}) \mathrm{k}=0,1,2, \ldots$

Where: when 3.571448 $<\mu<4$ [4], the Chaotic mapping is in Chaotic state. 3.571448< $<<4$ is called Chaotic area, $\mathrm{a} 0 \in(0,1)$, so Logistic mapping can be delimited to $(0,1)$. As a result the chaotic system can gain a pseudorandom sequence $\left\{a_{k}\right\}_{k=0}^{\infty}$. Which is delimited to $(0,1)$.

With respect to image hiding, from the point of pixel, we interpolate linearly between the gray values of the corresponding pixels in the two image pictures aiming at hiding a picture quickly [5]. 
Mark the picture to be hidden as B , the model picture as A and the end picture as E. The sizes of those three pictures are written as SizeB, SizeA, SizeE, in which SizeA=SizeB=SizeE .

When hiding two same-sized pictures, we assure the gray values of the corresponding pixels $(\mathrm{i}, \mathrm{j})$ in them are marked as $A(i, j)$ and $B(i, j)$, where $A(i, j)$ is the pixel gray values a of the original picture at $(i, j)$, and $B(i, j)$ is the pixel gray values a of the model picture at $(i, j)$. Chaotic sequences that are produced by Chaotic system is $\{$ ai, $\mathrm{j}\}, \mathrm{i}=1,2, \cdots, \mathrm{M}, \mathrm{j}=1,2, \cdots, \mathrm{N}$, (the gray value above represent a value in I or Q), in which $\mathrm{M} \times \mathrm{N}$ means the size of the image. There for the equation which is used to interpolate between the two pixels in the chaotic sequences:

$E(i, j)=a i, j \times(B(i, j)-A(i, j))+A(i, j) 0<a i, j<1$

To achieve the better hiding effect, interpolation fusion coefficient ai, $\mathrm{j}$ is required to be close to 0 as much as possible. However, the value of chaotic sequences meets ai, $j \in(0,1)$. Therefore the equation is amended below:

$$
E(i, j)=\frac{1}{2^{n}} \cdot a i, j \times(B(i, j)-A(i, j))+A(i, j) 0<a i, j<1
$$

Where: $n$ equals integers, called hiding factor. When the value of $n$ is bigger, the hiding effect is better. According to the equation (10) the image hiding Algorithm can be gotten correspondingly as follows:

Step1: input image B to be ciphered and model image A, and meanwhile give the primary value of the chaotic sequences which is 0 and hiding factor $n$.

Step2: In line with the size of model image, utilize the equation (9) to produce the same-sized Chaotic sequence (ai, j), where $0<$ ai, $\mathrm{j}<1 \mathrm{i}=1,2, \cdots, \mathrm{M}, \mathrm{j}=1,2, \cdots, \mathrm{N}$.

Step3: utilize equation (10) and chaotic sequences (ai, j) to calculate the two images, thus getting the ciphered image E. About the recovery of the hiding images, we can obtain the corresponding equation to calculate $\mathrm{F}(\mathrm{i}, \mathrm{j})$ by the hiding equation $(10)$ :

$\mathrm{B}(\mathrm{i}, \mathrm{j})=\frac{2^{n}}{a_{i, j}} \times(\mathrm{E}(\mathrm{i}, \mathrm{j})-\mathrm{A}(\mathrm{i}, \mathrm{j}))+\mathrm{A}(\mathrm{i}, \mathrm{j}), 0<\mathrm{ai}, \mathrm{j}<1$

We also can get the corresponding recovery Algorithm of hiding images.

The third step deals with Y component focusing on four upgrading wavelet decomposition , adopting energy method for the part of high frequency and employing averaging method fusing to get the intermediate quantity for the part of low frequency. Then handle the intermediate quantity and the Y component of two original images dividedly, and compare original image with average error MAE of every part of MY. The definition of average error:

$$
\begin{aligned}
M A E^{A} & =\frac{1}{M N} \sum_{i=1}^{M} \sum_{j=1}^{N}|M Y(i, j)-A Y(i, j)| \\
M A E^{B} & =\frac{1}{M N} \sum_{i=1}^{M} \sum_{j=1}^{N}|M Y(i, j)-B Y(i, j)|
\end{aligned}
$$

Where: M stands for linage and $\mathrm{N}$ for row: MY A Y BY are feedback sub block and A, B original sub blocks. We take the sub block whose difference of average error is smaller as fusion result, get the feedback quantity RY and feedback it to MY, readjust the size of whole block, and finally gain optimization fusion result FY of Y component . Its process is showed by Fig.1:

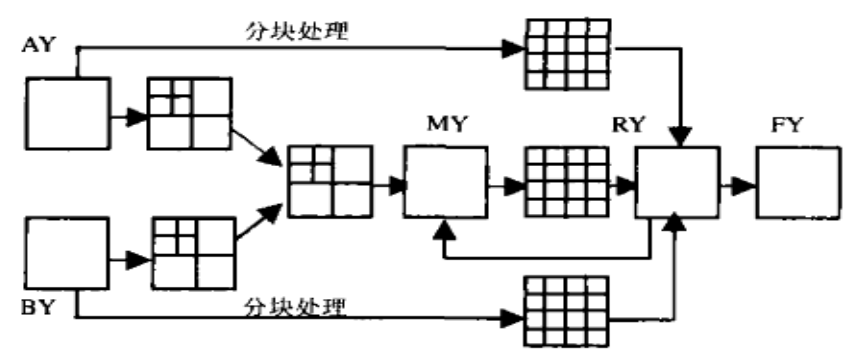

Fig. 1 The fusion process of Y component

Step4: The final fusion image can be obtained after transforming YIQ three components which have been fused to RGB space. 


\section{Experimental Simulations and Result Analysis}

A detection to the no attack of the hidden image

In the experiment, standard color test image (a) and virtual color multi-focus image (b) are adopted to do the experiment which fuses and hides color digital image.

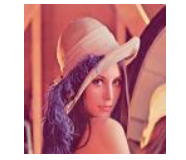

(a) Model image

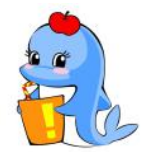

(b) Hiding image

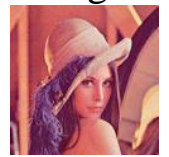

(c) Result image

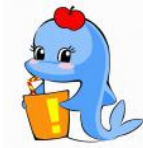

(d) Recovery image

Fig. 2 The image restoration and hide the image

Due to the limits of human visual ability to distinguish, objective assessment standard will be used to evaluate. Experimental function evaluation can be seen in table 1.

Table1 Fusion result evaluation chart of the experiment

\begin{tabular}{cccc}
\hline & H & RSME & PSNR \\
\hline Maximum method & 7.2451 & 7.1693 & 31.8964 \\
Contrast method & 7.2968 & 3.5674 & 40.5372 \\
Method of the passage & 7.3056 & 2.1867 & 47.1928 \\
\hline
\end{tabular}

Detection of the hidden image under attack

(1) JPEG compression. After doing compression experiment on resulting image (c) according to different quality parameters, the results are showed as table 2. And Fig. 3 shows the results when the quality parameter is 45 , in which case the quality of images has been lowered obviously, but the hidden images still be restored. In conclusion, the algorithm has perfect features of JPEG compress proof.

Table 2 Experiment results of the restored hidden images under JPEG compression attack.

\begin{tabular}{ccc}
\hline Quality parameters of JPEG compression /\% & NC & PSNR/dB \\
\hline 100 & 0.9998 & 43.51 \\
85 & 0.9416 & 41.63 \\
70 & 0.9203 & 40.76 \\
60 & 0.8461 & 35.45 \\
45 & 0.7389 & 32.19 \\
\hline
\end{tabular}

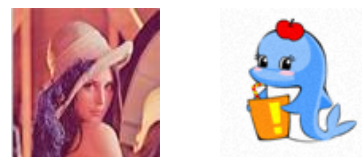

Fig. 3 The experiment results of JPEG compression

(2) Superimposed noise. The superimposed mean value of this experiment to resulting image [Fig.2(c)] is 0, and variance of Gaussian noise is 0.05. The results are as Fig.4. Here, the PSNR of blue components is $30.24 \mathrm{~dB}$. The NC between the restored hidden images and hidden images is 0.6071. Though low is the NC, people still can recognize them, for the restored hidden images are pictures.
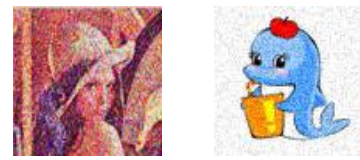

Fig. 4 The experiment results of superimposed Gaussian noise

(3) Cut procession in this experiment, after the resulting images [Fig.2(c)] are cut by 1/2, the cut parts are substituted by the original images. The hidden images can be still restored correctly. The results are as Fig. 5.

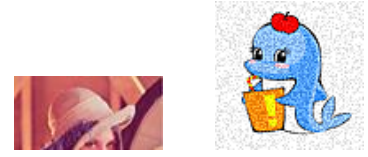

Fig. 5 The results of cut procession 


\section{Summary}

This passage raises a method which exploits the method of image fusion combining chaotic sequences, based on lifting wavelet transform, to hide color digital image. It also has dealt with the same-sized model image and hiding image with the algorithm successfully. The experimental results indicate that the image hiding algorithm given possesses the features of widely use and safety. What's more, the method is provided with better hiding and recovery effect.

\section{References}

[1]. Guo Lixin. Study on the characteristic of IR radiation from space target [A]. SPIE [C], 2004, 25 (1): 119-127.

[2]. Liu Changzheng Huang Maocheng. Fast Lifting Wavelet Transform Research and Implementation [J]. Microsystems Computer Information, 2009, 9 - 332 - 34.

[3]. Dong Weijun, torture people, and so on. Wavelet transform based on lifting scheme color digital watermarking technology [J]. Journal of Northwest University (Natural Science Edition), April 2006 Vol.36 No.2: 201-204.

[4]. Chen $\mathrm{Du}, \mathrm{Xu}$ Xiufang, Liu Yin, the other space exploration infrared spectroscopy signal processing techniques [J]. Infrared Technology, 2006, 28 (4), 203-206

[5]. Fei Synopsys R \& D center ed. MATALB 7 wavelet analysis theory and realization of [M]. Beijing: Electronic Industry Press, 2005.3: 275-281. 